Portland State University

PDXScholar

\title{
A compact method for optical induction of proximal probe heating and elongation
}

\author{
Andres H. La Rosa \\ Portland State University, andres@pdx.edu \\ Hans D. Hallen \\ North Carolina State University at Raleigh
}

Follow this and additional works at: https://pdxscholar.library.pdx.edu/phy_fac

Part of the Physics Commons

Let us know how access to this document benefits you.

\section{Citation Details}

A. H. La Rosa and H. Hallen, "A compact method for optical induction of proximal probe heating and elongation", Appl. Optics 41, 2015 (2002)

This Article is brought to you for free and open access. It has been accepted for inclusion in Physics Faculty Publications and Presentations by an authorized administrator of PDXScholar. Please contact us if we can make this document more accessible: pdxscholar@pdx.edu. 


\title{
Compact method for optical induction of proximal probe heating and elongation
}

\author{
Andres H. La Rosa and Hans D. Hallen
}

\begin{abstract}
A tapered, metal-coated, optical fiber probe will elongate when heated by light input through a fiber. The induced motion can be used for data storage or nanostructuring of a surface. The elongation produced by this alignment-free system is measured with force feedback in a near-field scanning optical microscope (NSOM). The input light intensity controls the elongation magnitude, which ranges from a few nanometers to more than $100 \mathrm{~nm}$. A $0.5-\mathrm{mW}$ input energy yields $\sim 20 \mathrm{~nm}$ of probe elongation. The elongation quantified here can create artifacts in any experiment using pulsed laser light with a NSOM or an atomic force microscope. (C) 2002 Optical Society of America
\end{abstract}

OCIS codes: $\quad 180.5810,350.1820,230.4000,260.2160,260.3910,260.3060$.

\section{Introduction}

Scanning proximal probes enable many types of nanoscale fabrication. Atoms can be moved one at a time to build surface structures with a scanning tunneling microscope (STM). ${ }^{1-3}$ Current bursts with a STM can create surface structures. ${ }^{4}$ Electrons injected from a STM into a metal thin film can produce terraces on the buried surface. ${ }^{5,6}$ The STM can be used as an electron source for electron-beam lithography, ${ }^{7}$ for electron-assisted chemical vapor deposition, 8 or to remove a surface layer. ${ }^{9}$ Near-field scanning optical microscopes (NSOMs) have been used to write magnetic bits;10 to expose resists; 11 to oxidize conjugated-polymer thin films; ${ }^{12}$ and, in combination with a current from the metal NSOM aperture, to locally modify the properties of high-temperature superconductor $\mathrm{YBa}_{2} \mathrm{Cu}_{3} \mathrm{O}_{7-\delta}$ (yttrium, barium, copper, oxygen). ${ }^{13}$ Atomic force microscopes (AFMs) have been used as dip pens for direct-write lithography ${ }^{14}$ and for writing bits into a substrate. ${ }^{15}$ A thermomechanical writing technique, ${ }^{16}$ by which optical pulses are coupled to a metallized tip of a tapered optical fiber, has been used in data-storage applications, yielding areal densities greater than 10 Gbits/in ${ }^{2}$. In this pa-

A. H. La Rosa (andres@pdx.edu) is with the Department of Physics, Portland State University, Portland, Oregon 97207-0751. H. D. Hallen is with the Department of Physics, North Carolina State University, Raleigh, North Carolina 27695-8202.

Received 17 November 2000; revised manuscript received 16 November 2001.

0003-6935/02/102015-05\$15.00/0

(C) 2002 Optical Society of America per we highlight that the thermomechanical technique can be extended to most scanning probe platforms and should be usable without head-stage modification in fiber-based NSOM systems. No special alignments will be required at the microscope head. The probe can be constructed so that none of the light reaches the sample ${ }^{17}$ when light absorption might alter the experiment. The thermal process at the probe is not characterized by a single time constant (in the 10-ms regime and associated mainly to the glass shank expansion) ${ }^{18,19}$ but includes those rapid time constants that result from expansion of the metal coating. This ensures reasonable speed performance of the elongation device. ${ }^{20}$ At the heart of a NSOM thermomechanical method is the probe axial elongation, which we analyze and quantify in this paper.

\section{Experiment}

The probe is similar to that used in the NSOM. It is constructed by heating and pulling of an optical fiber in a commercial apparatus and is then coated with aluminum. Metal coating is required, since the primary coupling of the light to heat the probe is by means of multiple imperfect reflections that occur when the light coupled into the core of the fiber reaches the tapered region of the probe. The absorption of light in the metal coating occurs mainly in the $\sim 100-\mu \mathrm{m}$ proximity of the probe apex, as predicted by ray-tracing-based models, ${ }^{21}$ and establishes a nonuniform axial temperature distribution. ${ }^{22}$ The probes used in this research had open apertures at the end, as NSOM probes do, but this is not required and makes little difference in the operation, since 
(a)

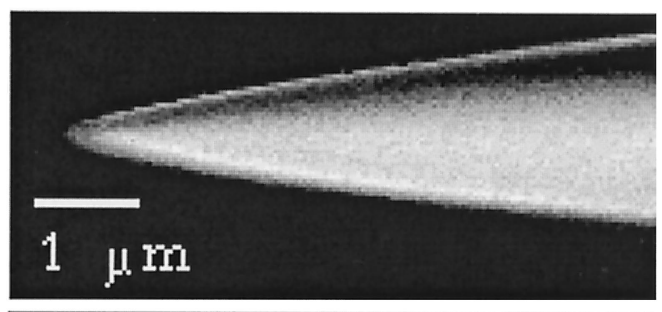

(b)

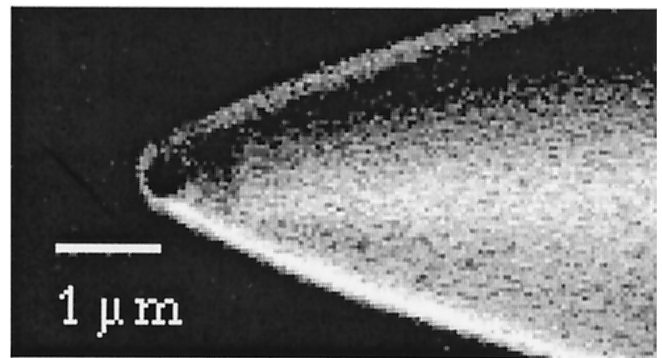

Fig. 1. SEM micrographs of typical uncoated (a) slender and (b) chubby tapered probe geometry used in the present experiment.

such a small fraction of the input light leaves the aperture. We studied the response of two different probe geometrical classes to illustrate the robustness of the probe response to probe morphology. Electron microscope (SEM) images of the two classes of probes are shown in Fig. 1. The chubby tip has a large aperture and a cone angle of $40 \mathrm{deg}$. The skinny tip has a smaller aperture and a 20 -deg cone angle near the apex. Both types of tip have similar structure far from the apex. The fiber tapers over a length of approximately $1 \mathrm{~mm}$.

The experimental layout is shown in Fig. 2. An acousto-optic modulator switches light from a laser, which is then coupled into the probe fiber. Several

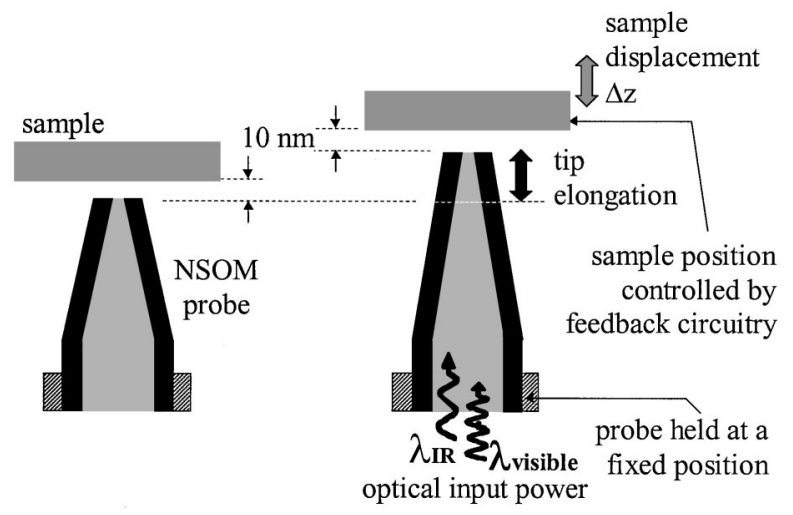

(a)

(b)

Fig. 2. (a) Tip is placed at a fixed lateral position relative to the sample, with no light coupled into the probe. By use of the shear force mechanism ${ }^{23}$ (not shown in the figure), the probe-sample distance is maintained at approximately $10 \mathrm{~nm}$. (b) When modulated visible light (or IR light) couples into the probe, the probe elongates and contracts, causing the shear force mechanism of the NSOM to respond. The tip-sample distance remains fixed, and we record the resulting up and down sample displacements. A typical modulation intensity for the visible or near-infrared input

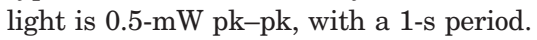

modulations were used, from blocking the beam manually to square-wave modulation between 1 and 10 $\mathrm{Hz}$. We used visible (512-nm green from an argonion laser) and infrared (1.15 $\mu \mathrm{m}$ from a He-Ne laser) light, with oN powers of $\sim 0.5 \mathrm{~mW}$. The probe is mounted in a NSOM. The microscope holds the tip in a fixed position of $\sim 10 \mathrm{~nm}$ over a flat sapphire or silicon sample, without scanning the tip. The lateral force feedback maintains a constant tip-sample separation. Thus the motion of the sample reflects the compensation for the probe elongation. Optical leverarm amplification of the probe motion, measured by a properly placed edge of a photodiode detector, provides the signal for lateral force detection. ${ }^{23}$ The bandwidth of the lateral force detection scheme limited the temporal range over which probe elongation could be measured. When the time scale of the probe elongation is too small for the feedback to follow, the probe deviates from its $\sim 10$-nm fixed distance from the surface. If the probe motion is fast and large enough, the probe will crash into the surface. This is the primary reason for using low-frequency modulation and only $0.5 \mathrm{~mW}$ of power, which gives approximately $20 \mathrm{~nm}$ of probe motion, although we expect elongation dependence on the quality of the aluminum coating. For square-wave light motion, inducing square-wave probe elongation, the feedback will slew at its bandwidth of $>1 \mathrm{kHz}$ to return the probe-sample separation to its nominal value. Some transient error in positioning may result after the light switches.

\section{Results and Discussion}

Examples of the measurements for a slender probe are shown in Fig. 3. The nominal input power level of infrared and visible light is $0.5 \mathrm{~mW}$ in both cases. Note the correlation between input power and the probe elongation, regardless of the wavelength. Although the data shown in Fig. 3(a) would suggest that elongations produced by the infrared light are greater than the ones produced by the visible light, in Fig. 3(b) the opposite occurs. This is attributable to the fact that the actual power reaching the probe depends on many factors such as losses along the fiber and different coupling efficiency for infrared and for visible light. The probe fiber is few mode for the visible light, so the probe transmission is a strong function of the mode traveling in the fiber, i.e., the coupling into and the positioning of the fiber. We understand that the probe elongation will be more sensitive to the energy input rather than to the particular wavelength chosen because the probe elongation relies on heating and because the large number of reflections within the probe ${ }^{21}$ ensures that a significant fraction of any wavelength light is absorbed by imperfect reflections from the metal coating on the probe taper. The shape of the taper resembles a small version of a witch's hat beam dump.

To determine whether significant thermal energy is transferred to the sample when it is in close proximity to the sample, the elongation was measured as a function of probe-sample distance, shown in Fig. 4. On the length scales of interest here, thermal con- 


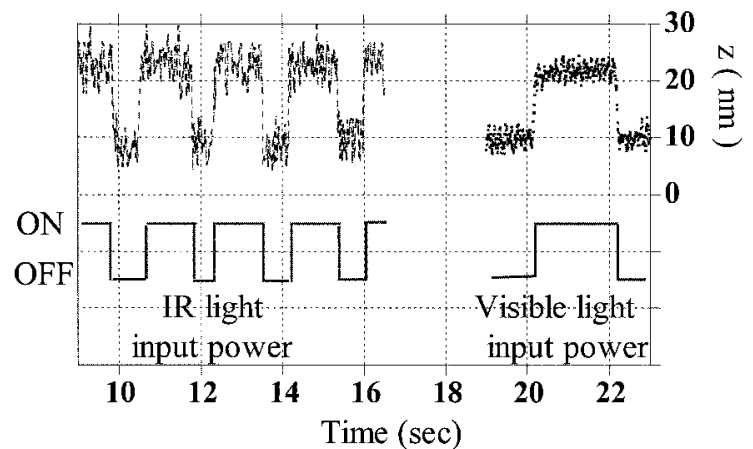

(a)

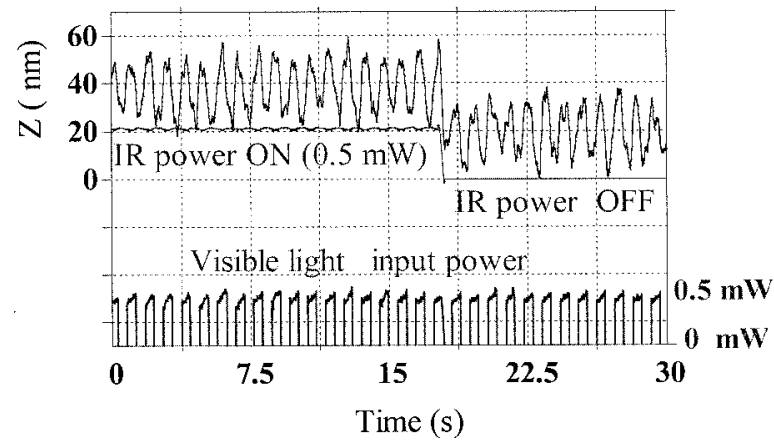

(b)

Fig. 3. (a) Probe elongation as a function of time is shown (left) driven by IR light and (right) driven by visible light. Both input powers were blocked manually. The sapphire-sample displacement follows the elongation and contraction of the probe. The nominal value of the input power in each case is $0.5 \mathrm{~mW}$; the corresponding different elongations they produce on the probe result from the uncertainties of the actual input power reaching the probe. (b) (Modulated) visible and (continuous-wave) IR input powers correlate with tip elongation. The sample (silicon) displacement $Z$ follows the elongation/contraction of the probe. In the oN state, both input powers have a nominal value of $0.5 \mathrm{~mW}$. Bottom curve, modulated input visible light; top curve, elongation of the probe. Note that the probe experiences an additional elongation when the infrared beam is turned on.

duction would be the prime mode of heat transfer. ${ }^{24}$ If significant energy flowed from the tip to the sample, one would expect the probe elongation to decrease as the separation does. No clear trend exists in the data, suggesting that the heat transfer is negligible. This result is expected, since most NSOM measurements, including those on cells, ${ }^{25,26}$ have not found any detectable damage caused by heating. Liquid crystals represent one of the few samples for which thermal damage has been indicated. ${ }^{27}$

The modulation level is determined by the input power. We are somewhat limited in the range of powers that we can study with force feedback to measure the probe elongation, since the probe may transiently crash into the sample. Alternatively, one can use the Fabry-Perot cavity formed by a flat surface and the NSOM-probe flat termination to estimate the probe elongation ${ }^{28}$ and thus avoid the limited bandwidth feedback response, which is described in more detail elsewhere. ${ }^{29}$ A probe coated

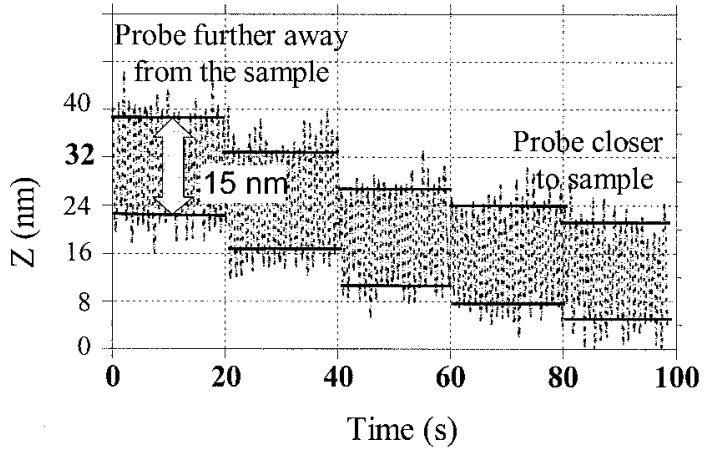

Fig. 4. Measurement of the probe elongation and contraction for different probe sample distances. The light is modulated at $1 \mathrm{~Hz}$ producing rapid sample positioning variations. Reduction of the feedback level in steps causes a reduction in the probe-sample distance. From left to right, probe-sample distance decreases in discrete steps; however, the probe elongation remains approximately invariant (a 15-nm window is shown for comparison). Typically, the feedback stability in our system is $1 \mathrm{~nm}$. Data outside the 15-nm window result from instabilities of the input power laser.

with aluminum will degrade if the temperature reaches half the melting temperature of aluminum, or $\sim 470 \mathrm{~K}$, when multiple internal reflections, diffusion, and surface energy combine to make the aluminum ball up. Using this as a limit, we estimate the maximum elongation of the probe to be $\sim 200 \mathrm{~nm}$, at 4.5-mW input power.

We previously studied the temporal optical throughput response of the classes of probes used here, both before and after probe damage. ${ }^{18}$ Those results indicate a response independent of frequency to $\sim 20 \mathrm{~Hz}$. At higher frequencies, the response falls logarithmically. The process cannot be modeled as a single-time-constant response, which would fall as $1 / f$. This is common to many thermal problems. It means that the probe elongation is still present at higher frequencies and that this probe elongation method is applicable for fast probe motion. The amplitude will be $1 / 5$ its low-frequency value at $10 \mathrm{kHz}$. The slow variation of the optical throughput implies that a significant fraction of the taper is heated $(L \sim$ $200 \mu \mathrm{m}),{ }^{18}$ which causes these slow-motion states to be associated with the bigger contribution to the probe elongation $\Delta L$. The faster-motion states, described by shorter thermal time constants, are then associated to much smaller regions near the probe apex. In this regard, it is worth noting that the rainbow of thermal time constants available in NSOM-like probes contains the fast time constants reported in thermomechanical writing processes with AFM probes. ${ }^{30,31}$ AFM probes lack, however, the slow time constant because they lack the shank present in NSOM-like probes, which, in addition, provides a channel for heat dissipation and better protects the tip. The slowest time constants do not matter in data writing as long as the bits are written fast enough that the probe elongation stays in a dynamic equilibrium value by either (a) the tip not be- 


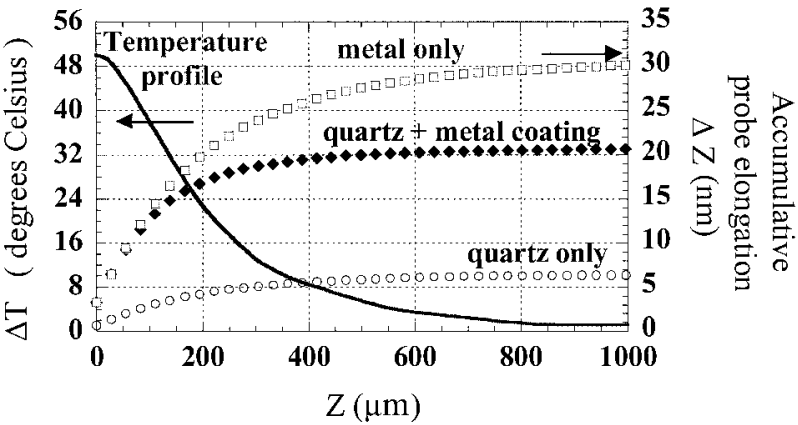

Fig. 5. Temperature along the probe is not uniform, as shown by the solid curve (adapted from the experimental data in Ref. 22). A temperature increase of $50{ }^{\circ} \mathrm{C}$ at the tip end is expected for a $0.5-\mathrm{mW}$ input power. The temperature profile is used to calculate the thermal expansion of a 1-mm-long bare fiber (circles) and a full metal probe (squares). The aluminum coating is taken into account by considering a position-dependent thermal coefficient that equals $\alpha_{\mathrm{AL}}$ at the tip end and $\alpha_{\text {quartz }}$ at the shank. The corresponding probe expansion (diamonds) provides a better fit to the observed probe thermal expansion in our experiment. Note that the region closer to the tip end contributes more to the elongation.

ing allowed to heat or cool by amounts that vary on that time scale or (b) running a slow feedback loop to compensate.

Because the temperature distribution along the axial direction of the probe is not uniform, the contribution to the total probe elongation from the apex region can be more significant than the contribution from the probe shank. Using temperature profiles obtained by M. Stahelin et al., ${ }^{22}$ as well as previous estimates by us ${ }^{18}$ and others ${ }^{32}$ dealing with temperature rise of NSOM probes, we have estimated the contribution to the probe elongation from the different sections of a NSOM probe. This is shown in Fig. 5. According to this temperature profile, a 1-mmlong bare fiber (coefficient of thermal expansion $\alpha_{\text {quartz }}=0.5 \times 10^{-6} / \mathrm{K}$ ) will contribute only $6 \mathrm{~nm}$ to the total elongation. Had we considered pure aluminum $\left(\alpha_{\mathrm{Al}}=2.35 \times 10^{-6} / \mathrm{K}\right)$, we would have obtained 30-nm probe elongation, which is in excess of what we typically observe in our probes. The latter case does not take into account that there exists a high concentration of aluminum only at the apex region, whereas the back side is dominated by quartz. For that reason we have introduced, in the thermal expansion calculation, a weighting factor proportional to the local temperature along the probe, which reflects the bigger role played by the aluminum coating only at the tip-end region. We use $\alpha_{\text {effective }}(z)=$ $\alpha_{\text {quartz }}+\beta * 2 a \Delta T(z)$, where $\beta$ is chosen such that $\alpha_{\text {effective }}=\alpha_{\mathrm{Al}}$ at $z=0$. For large values of $z, \Delta T$ is small and $\alpha_{\text {effective }}$ becomes equal to $\alpha_{\text {quartz. }}$. With this correction in the thermal expansion coefficient, we calculate $20-\mathrm{nm}$ probe elongation when the temperature at the tip end rises by $50{ }^{\circ} \mathrm{C}$, which is much closer to the typical thermal elongation we measure.

The layered structure, or metal-coated silica, of the NSOM probe can cause the probe end to have a different time response than the rest of the probe (the tapered region). Indeed, numerical analysis of a truncated-cone geometry indicates that a metallic tip would have a very fast response (approximately microseconds). ${ }^{20}$ Recently, tip response faster than the $15 \mathrm{~ms}$ associated with optical throughput variation has been reported. ${ }^{19}$ The metal occupies a larger fraction of the cross-sectional area near the tip than the silica does. It conducts heat much faster, resulting in faster thermal time constants. The aluminum also expands more than the glass with temperature (Fig. 5). We therefore envision a rapid sliding of the metal layer over (or distortion of) the silica layer near the tip. The slower response of the tip shank, dominated by the silica, accounts for the remainder of the expansion.

Although we quantify the effect for apertured NSOM here, the elongation will also be present in apertureless NSOM experiments. The effects described here are important to any experiment that uses light of varying intensity, such as pulsed lasers, in a scanning probe (AFM, NSOM, and so on) experiment. It is not possible to avoid this probe heating response. Applications of this method include data storage and other applications in which probe motion normal to the surface is required.

The NSOM thermomechanical method described here does not require any alignment on the microscope head. It does require the light to be coupled into the probe fiber, but this can be accomplished in a one-time light-to-fiber coupling and subsequent routine use of a butt coupler. This method also does not expose the sample to light, although it is probably not an issue for this particular application. Further, NSOM probes have been found to be fairly robust to wear, with wear of the metal aperture being the primary weak point. Since the probe-elongation method described here operates without dependence on the properties of the tip of the probe, it should be robust against wear. Since tip wear and replacement would be a part of any such system in actual use, our method would provide a suitable choice for manufacturing.

In conclusion, we have demonstrated that probe elongation can be driven by optical power input to a NSOM-like probe. The elongation is independent of the wavelength used and the distance from the sample. The frequency response and properties suggesting its use for delivery of heat or mechanical impulses to a surface have been described. We note that the process quantified here should be taken into account whenever pulsed light is used in NSOM or AFM experiments.

A. H. La Rosa acknowledges grant start-up funding from Portland State University.

\section{References}

1. D. M. Eigler and E. K. Schweizer, "Positioning single atoms with a scanning tunneling microscope," Nature 344, 524-526 (1990).

2. Ph. Avouris, I.-W. Lyo, and Y. Hasegawa, "Scanning tunneling microscope tip-sample interactions: atomic modification of $\mathrm{Si}$ 
and nanometer Si Schottky diodes,” J. Vac. Sci. Technol. A 11, 1725-1732 (1993).

3. L. J. Whitman, J. A. Stroscio, R. A. Dragoset, and R. J. Celotta, "Manipulation of adsorbed atoms and creation of new structures on room-temperature surfaces with a scanning tunneling microscope," Science 251, 1206-1210 (1991).

4. H. J. Mamin, P. H. Guethner, and D. Rugar, "Atomic emission from a gold scanning-tunneling-microscope tip," Phys. Rev. Lett. 65, 2418-2421 (1990).

5. H. D. Hallen, A. Fernandez, T. Huang, R. A. Buhrman, and J. Silcox, "Gold-silicon interface modification studies," J. Vac. Sci. Technol. B 9, 585-589 (1991).

6. H. D. Hallen, A. Fernandez, T. Huang, R. A. Buhrman, and J. Silcox, "Hot electron interactions at the passivated gold-silicon interface," Phys. Rev. Lett. 69, 2931-2934 (1992).

7. C. R. K. Marrian, E. A. Dobisz, and John A. Dagata, "Electronbeam lithography with the scanning tunneling microscope," J. Vac. Sci. Technol. B 10, 2877-2881 (1992).

8. S. Rubel, M. Trochet, E. E. Ehrichs, W. F. Smith, and A. L. de Lozanne, "Nanofabrication and rapid imaging with a scanning tunneling microscope,” J. Vac. Sci. Technol. B 12, 1894-1897 (1994).

9. R. S. Becker, G. S. Higashi, Y. J. Chabel, and A. J. Becker, "Atomic-scale conversion of clean $\mathrm{Si}(111): \mathrm{H}-1 \times 1$ to $\mathrm{Si}(111)$ $2 \times 1$ by electron-stimulated desorption," Phys. Rev. Lett. 65, 1917-1920 (1990).

10. E. Betzig, J. K. Trautman, R. Wolfe, E. M. Gyorgy, and P. L. Finn, "Near-field magneto-optics and high density data storage," Appl. Phys. Lett. 61, 142-144 (1992).

11. S. Madsen, M. Müllenborn, K. Birkelund, and F. Grey, "Optical near-field lithography on hydrogen-passivated silicon surfaces," Appl. Phys. Lett. 69, 544-546 (1996).

12. P. K Wei, R. Hang, J. H. Hsu, S. H. Lin, W. S. Fann, and B. R. Hsieh, "Two-dimensional near-field intensity distribution of tapered fiber probes," Opt. Lett. 21, 1876-1878 (1996).

13. S. H. Huerth, M. P. Taylor, H. D. Hallen, and B. H. Moeckly, "Electromigration in YBCO using a metal clad near-field scanning optical microscope probe,” Appl. Phys. Lett. 77, 21272129 (2000).

14. S. Hong and C. A. Mirkin, "A nanoplotter with both parallel and serial writing capabilities," Science 288, 1808-1811 (2000).

15. H. J. Mamin and D. Rugar, "Thermomechanical writing with an atomic force microscope tip," Appl. Phys. Lett. 61, 10031005 (1992).

16. S. Hoen, H. J. Mamin, and D. Rugar, "Thermomechanical data storage using a fiber optic stylus," Appl. Phys. Lett. 64, 267269 (1994).

17. D. Zeisel, S. Nettesheim, B. Dutoit, and R. Zenobi, "Pulsed laser-induced desorption and optical imaging on a nanometer scale with scanning near-field microscopy using chemically etched fiber tips," Appl. Phys. Lett. 68, 2491-2492 (1996).
18. A. H. La Rosa, B. I. Yakobson, and H. D. Hallen, "Origins and effects of thermal processes on near-field optical probes," Appl. Phys. Lett. 67, 2597-2599 (1995).

19. P. G. Gucciardi, M. Colocci, M. Labardi, and M. Allegrini, "Thermal-expansion effects in near-field optical microscopy fiber probes induced by laser light absorption,” Appl. Phys. Lett 75, 3408-3410 (1999).

20. See, for example, related studies on thermal effects in truncated cone metallic tips: V. Gerstner, A. Thon, and W. Pfeiffer, "Thermal effects in pulsed laser assisted scanning tunneling microscopy," J. Appl. Phys. 87, 2574-2580 (2000).

21. B. I. Yakobson, A. La Rosa, H. D. Hallen, and M. A. Paesler, "Thermal/optical effect in NSOM probes," Ultramicroscopy 61, 179-185 (1995).

22. M. Stähelin, M. A. Bopp, G. Tarrach, A. J. Meixner, and I. Zschokke-Gränacher, "Temperature profile of fiber tips used in scanning near-field optical microscopy," Appl. Phys. Lett. 68, 2603-2605 (1996).

23. M. A. Paesler and P. J. Moyer, Near-Field Optics: Theory, Instrumentation and Applications (Wiley, New York, 1996).

24. J.-B. Xu, K. Lauger, R. Moller, K. Dransfeld, and I. H. Wilson, "Heat transfer between two metallic surfaces at small distances,” J. Appl. Phys. 76, 7209-7216 (1994).

25. E. Betzig, R. J. Chichester, F. Lanni, and D. L. Taylor, "Nearfield fluorescence imaging of cytoskeletal actin," Bioimaging $\mathbf{1}$, 129-135 (1993).

26. J. Hwang, L. K. Tamm, C. Böhm, T. S. Ramalingam, E. Betzig, and M. Edidin, "Nanoscale complexity of phospholipid monolayers investigated by near-field scanning optical microscopy," Science 270, 610-614 (1995).

27. P. J. Moyer, K. Walzer, and M. Hietschold, "Modification of the optical properties of liquid crystals using near-field scanning optical microscopy," Appl. Phys. Lett. 67, 2129-2131 (1995).

28. A. La Rosa and H. D. Hallen, "Heat effects on the performance of near-field scanning optical microscopy probes as FabryPerot mirrors," presented at the American Physical Society Northwest Section Annual Meeting, Eugene, Oreg., 19-20 May 2000.

29. B. Biehler, "Characterization of thermal probe elongation in near-field optical microscopy," M.S. thesis (Physics Department, Portland State University, Portland, Oreg., 2001).

30. G. Binnig, M. Despont, U. Drechsler, W. Hberle, M. Lutwyche, P. Vettiger, H. J. Mamin, B. W. Chui, and T. W. Kenny, "Ultrahigh-density atomic force microscopy data storage with erase capability,” Appl. Phys. Lett. 74, 1329-1331 (1999).

31. M. I. Lutwyche, M. Despont, U. Drechsler, U. Drig, W. Hberle, H. Rothuizen, R. Stutz, R. Widmer, G. K. Binnig, and P. Vettiger, "Highly parallel data storage system based on scanning probe arrays," Appl. Phys. Lett. 77, 3299-3301 (2000).

32. D. I. Kavaldjiev, R. Toledo-Crow, and M. Vaez-Iravani, "On the heating of the fiber tip in a near-field scanning optical microscope," Appl. Phys. Lett. 67, 2771-2773 (1995). 